\title{
Tentativas de suicídio e mortalidade por suicídio em um município no interior da Bahia
}

\author{
Suicide attempts and suicide mortality in a countryside Bahia city \\ Viviane dos Santos Souza', Murilo da Silva Alves', Lívia Angeli Silva², Débora Cristiane Silva Flores Lino³, \\ Adriana Alves Nery 4 , Cezar Augusto Casotti ${ }^{4}$
}

\section{RESUMO}

Objetivo: Descrever o perfil das tentativas de suicídio e mortalidade por suicídio no município de Jequié/BA, no período entre 2006 e 2010. Método: Estudo epidemiológico descritivo. Os dados das tentativas de suicídio foram obtidos no Corpo de Bombeiros e DATASUS, enquanto aqueles referentes à mortalidade por suicídio foram coletados por meio das declarações de óbito (DOs) da 13a Diretoria Regional de Saúde da Bahia (13a DIRES) e complementados pelos registros do Instituto Médico Legal (IML). A análise foi feita por meio de estatística descritiva. Resultados: Nas tentativas de suicídio, a maior frequência foi na faixa etária de 20 a 39 anos (57,69\%), entre indivíduos do sexo feminino (53,4\%) e utilizando-se como meio a queda de altura (69,2\%). Em relação ao suicídio, a taxa média de mortalidade foi de 3,19/100.000 habitantes, com maior frequência em indivíduos acima dos 40 anos (54,16\%), do sexo masculino (87,5\%), por meio de enforcamento $(58,3 \%)$ e tendo como local de ocorrência

\section{Palavras-chave}

Suicídio, tentativa de suicídio, epidemiologia, declaração de óbito.

\section{Keywords}

Suicide, suicide attempt, epidemiology, death certificate. o domicílio (83,33\%). Conclusão: O município mostrou-se dentro das estatísticas da região Nordeste e abaixo das taxas nacionais de suicídio. Houve diferenciação entre as características do perfil das tentativas de suicídio e mortalidade por suicídio, especialmente segundo o sexo, faixa etária e meios utilizados.

\section{ABSTRACT}

Objective: Describing the profile of suicide attempts and suicide mortality in the city of Jequié/BA, in the period between 2006 and 2010. Method: A descriptive epidemiological study. Data of suicide attempts were obtained from the Fire Department and DATASUS, while those related to suicide mortality were collected by means of Death Certificates (DOs) of the $13^{\text {th }}$ Regional Health Board of Bahia (13 $3^{\text {th }}$ DIRES) and supplemented by records from the Forensic Medicine Institute (IML). The analysis was made using descriptive statistics. Results: In suicide attempts, the biggest frequency was in people aged from 20 to 39 years (57.69\%); among females (53.4\%) and using as means the fall from height (69.2\%). Regarding suicide, the average mortality rate was 3.19/100,000 inhabitants, most frequently in people over 40 years (54.16\%), male (87.5\%), by hanging (58.3\%) and taking the place of occurrence as the home (83.33\%). Conclusion: The city showed up in the statistics of the Northeast region and below the national rates of suicide. There was differentiation between the profile characteristics of suicide attempts and suicide mortality, especially by gender, age and used means.

1 Universidade Estadual do Sudoeste da Bahia (UESB), Programa de Pós-Graduação em Enfermagem e Saúde (PPGES).

2 UESB, Instituto Federal de Educação, Ciência e Tecnologia da Bahia (IFBA), PPGES.

3 Universidade Estadual de Santa Cruz (UESC), Grupo de Pesquisa em Saúde Mental.

4 UESB, Departamento de Saúde, PPGES.

Endereço para correspondência: Universidade Estadual do Sudoeste da Bahia (UESB), Programa de Pós-Graduação em Enfermagem e Saúde (PPGES). Rua José Moreira Sobrinho, s/n, Jequiezinho - 45206-190 - Jequié, BA

Telefax: (73) 3528-9738

E-mail: ppgesjq@uesb.edu.br 


\section{INTRODUÇÃO}

O suicídio constitui-se como um grave problema de saúde pública mundial, em especial por seu crescimento entre a população mais jovem ${ }^{1,2}$. Segundo estimativas da Organização Mundial de Saúde (OMS), o suicídio é definido como o ato humano de causar a cessação da própria vida, sendo uma das dez principais causas de morte em todo o mundo, chegando a ocupar a terceira posição entre os óbitos ocorridos na faixa etária de 15 a 35 anos 3,4 .

As tentativas de suicídio e o comportamento suicida podem ser conceituados como atos intencionais de autoagressão que não resultam em morte, podendo envolver atos mais graves, para os quais são necessárias hospitalizações. Algumas autoagressões podem, ainda, não necessitar de atendimento médico, o que dificulta a realização de estudos acerca das tentativas de suicídio ${ }^{5}$.

Dados da OMS mostram que, no ano de 2000, 1 milhão de pessoas cometeram suicídio no mundo, o que representou uma morte a cada 40 segundos ${ }^{5,6}$. Anualmente, 10 a 20 milhões de pessoas tentam o suicídio7. O Brasil apresenta uma taxa geral de suicídio considerada baixa na escala mundial (4 a 6 óbitos por 100.000 habitantes), ocupando o $71^{\circ}$ lugar quando comparado com outros países no mundo e 9a posição em números absolutos de mortes por suicídio ${ }^{2,8}$.

O Brasil encontra-se distante das maiores taxas de suicídio mundiais, mais de 16/100.000 habitantes, como encontradas na França, China, Suíça, Bélgica, Áustria, Estados Unidos e no Leste Europeu. Contudo, registros nacionais sobre a mortalidade demonstram o aumento da incidência de suicídios na faixa etária de adulto jovem, principalmente no sexo masculino, mesmo considerando as possíveis subnotificações existentes $^{3,5,9}$. De acordo com dados de 2006, a mortalidade por suicídio no Brasil computou um total de 8.506 mortes, correspondendo a 6,7\% do total de mortes naquele ano ${ }^{3}$.

Entre as regiões brasileiras, a região Sudeste concentra $50 \%$ dos registros de suicídio; a região Sul possui os maiores coeficientes de suicídio; e as regiões Norte e Nordeste apresentam os menores índices. Os estados brasileiros com maiores taxas de suicídio são o Rio Grande do Sul (8 a 10/100.000 habitantes), Santa Catarina (7 a 8,5/100.000 habitantes), Paraná (7,1 suicídios por 100.000 habitantes), seguidos de São Paulo e Goiás ${ }^{7}, 10,11$,

A região Norte apresentou a média de 3,4 mortes por 100 mil/habitantes e a Nordeste, uma média de 2,7 mortes por $100 \mathrm{mil} /$ habitantes. Os maiores aumentos de suicídio foram verificados na região Nordeste, que experimentou um incremento de 130\% entre 1980 e 2006, e na região Centro-Oeste, com aumento de $68 \%$ no mesmo período ${ }^{12}$.

Diversos estudos comprovaram fatores associados ao suicídio, principalmente os socioeconômicos. Nesse sentido, a baixa escolaridade e a pobreza, normalmente associadas entre si, além de viuvez, perda de uma pessoa querida, sepa- ração afetiva, desentendimentos com familiares ou amigos, divórcio e solidão das grandes cidades, são considerados fatores ou circunstâncias que podem aumentar o risco de suicídio por serem produtores de estresse, bem como outros, que incluem religião, problemas legais, desemprego e trabalho, especialmente o agrícola ${ }^{3,4,8}$.

Uso abusivo de álcool e drogas, disponibilidade dos meios para efetuar o ato suicida, violência física e/ou sexual na infância, isolamento social, baixo suporte social e familiar, baixa qualificação profissional, histórico de tratamento psiquiátrico e distúrbios psíquicos, como depressão, esquizofrenia ou falta de esperança, podem, ainda, ser contributivos para o aumento de risco e a consumação da tentativa de suicídio 4,9,10,13.

Tais observações demonstram que esse ato não possui uma única causa ou razão, mas resulta de uma complexa interação de fatores, entre eles os biológicos, genéticos, psicológicos, sociais, culturais e ambientais, sendo difícil explicar como a exposição aos mesmos fatores de risco implicam condutas diferenciadas dependendo dos sujeitos envolvidos ${ }^{3,4,8}$.

O comportamento autodestrutivo, como o uso de substâncias psicoativas, recusa de tratamento médico, determinados estilos de vida que também podem resultar em morte, é considerado por alguns autores como comportamento suicida, mas que pode ser distorcido ou mascarado como se tivesse sido natural ${ }^{5}$.

O suicídio tem impactos diretos sobre a vida de familiares e pessoas próximas ao suicida. Além disso, representa prejuízos para a sociedade, pelos investimentos necessários para a formação do indivíduo, anos potenciais de vida perdidos devido à morte precoce e aos custos de internações com indivíduos que não obtêm sucesso com o ato. Na perspectiva de prevenir comportamentos suicidas, foi publicado em 2006, pelo Ministério da Saúde, o Manual de Prevenção ao Suicídio, destinado aos profissionais das equipes de saúde mental ${ }^{14}$.

No Brasil e em outros países, a qualidade dos dados disponibilizados referentes aos óbitos é fator que limita as pesquisas sobre a mortalidade por suicídio ${ }^{10}$. A ocorrência de subnotificação dos casos de suicídio acontece devido a motivações religiosas, sociais, culturais, entre outras ${ }^{15}$. Alguns autores indicam que essa distorção deve variar no espaço e no tempo, entre $20 \%$ e $100 \% 5$.

Tais distorções podem estar relacionadas a estigma social acerca do óbito por suicídio, razões jurídicas, religiosidade devido à não aprovação do suicídio e, ainda, a relutância ou preenchimento inadequado pelo profissional médico ao registrar o óbito como "morte acidental" ou "causa indeterminada"16.

Os acidentes e as violências têm causado intenso impacto na morbimortalidade da população brasileira. Por isso, o governo tem implementado políticas públicas, a exemplo da Política Nacional de Redução da Morbimortalidade por Acidentes e do Plano Nacional para Prevenção do Suicídio, lançado em 2009. Estas surgem como iniciativas do Ministério da Saúde para tentar diminuir a incidência de casos no 
Brasil, visando à redução das taxas de mortes por suicídio e danos causados às pessoas envolvidas direta e indiretamente nesse ato ${ }^{3}$.

Tais políticas são importantes, uma vez que a rede de saúde enfrenta desafios para desenvolver ações preventivas e assistenciais relacionadas ao suicídio. Nesse sentido, mais estudos são necessários, com o objetivo de produzir informações que colaborem na formulação de estratégias para a redução da morbimortalidade da população, reduzindo as taxas de tentativas de suicídio no Brasil.

Assim, visando melhorar a situação de saúde e subsidiar o planejamento de ações preventivas nos serviços de saúde, este estudo objetivou descrever o perfil das tentativas de suicídio e mortalidade por suicídio no município de Jequié/BA, no período de 2006 a 2010.

\section{MÉTODO}

Trata-se de estudo epidemiológico descritivo, com dados do Sistema de Informação de Mortalidade (SIM) obtidos na $13^{a}$ Diretoria Regional de Saúde da Bahia (13a DIRES) e do $8^{\circ} \mathrm{Gru}-$ pamento de Bombeiro Militar ( $8^{\circ} \mathrm{GBM}$ ) de Jequié/BA, complementados com dados do Instituto Médico Legal (IML) e do Sistema de Informação Hospitalar (SIH) do DATASUS.

O município de Jequié localiza-se na região Sudoeste da Bahia, distante 365 quilômetros de Salvador, capital do estado, e possui uma população de 151.921 habitantes $^{17}$.

Os dados de mortalidade foram obtidos na 13a DIRES, por meio das declarações de óbito (DOs) registradas no SIM, correspondentes às Causas Externas da Classificação Internacional de Doenças (CID-10). Quando necessário esclarecimento ou confirmação da causa de morte, uma vez que alguns campos da DO não estavam preenchidos ou remetiam à duplicidade de causa entre suicídio e homicídio, foram consultados os registros do IML do município.

Para identificar as tentativas de suicídio, recorreu-se aos dados do $8^{\circ} \mathrm{GBM}$ de Jequié/BA, por este possuir melhor qualidade de informações, uma vez que é o serviço requisitado para atendimento nesses casos. Para fins deste estudo, foram incluídas nessa categoria as ameaças de suicídio que não se concretizaram em virtude da intervenção desse serviço e que são registradas por ele. Os dados foram obtidos nas planilhas de controle de ocorrência, nas quais existe um campo específico para as tentativas de suicídio, sendo descartadas as que obtiveram sucesso, com óbito, uma vez que já estavam presentes nas DOs da 13a DIRES e IML.

Os dados foram analisados por meio de estatística descritiva, utilizando o programa Excel versão 2007. Foram definidas como variáveis para o suicídio: sexo, idade, etnia, escolaridade, estado civil, ocupação, local de ocorrência do evento, local de moradia, meio utilizado, CID-10, horário, dia da semana, mês e ano do óbito.
Em relação às tentativas, as variáveis foram em menor número, devido à pequena quantidade de informações disponibilizadas nos relatórios do Corpo de Bombeiros sobre as ocorrências. Assim, utilizaram-se as variáveis: sexo, idade, local de ocorrência do evento, local de moradia, meio utilizado, horário, dia da semana, mês, ano da ocorrência e consumação das tentativas. Analisou-se, ainda, a tendência das internações por tentativa de suicídio no período de 2006 a 2010, com base nos dados do SIH/DATASUS.

O projeto de pesquisa foi avaliado pelo Comitê de Ética em Pesquisa da Universidade Estadual do Sudoeste da Bahia (CEP/UESB), sendo aprovado com o parecer n²10/2010.

\section{RESULTADOS}

No período 2006 a 2010, foram registrados no município de Jequié 24 suicídios (taxa média de mortalidade de 3,19 por 100.000 habitantes) e 26 tentativas de suicídio, com média de 3,45 casos por 100.000 habitantes. Na distribuição ao longo dos cinco anos, há uma tendência decrescente nos casos de suicídio, apresentando um pico no ano de 2009 $(4,64 / 100.000)$ com a maior taxa do período. Em contrapartida, as tentativas apresentam uma tendência crescente, conforme a figura 1.

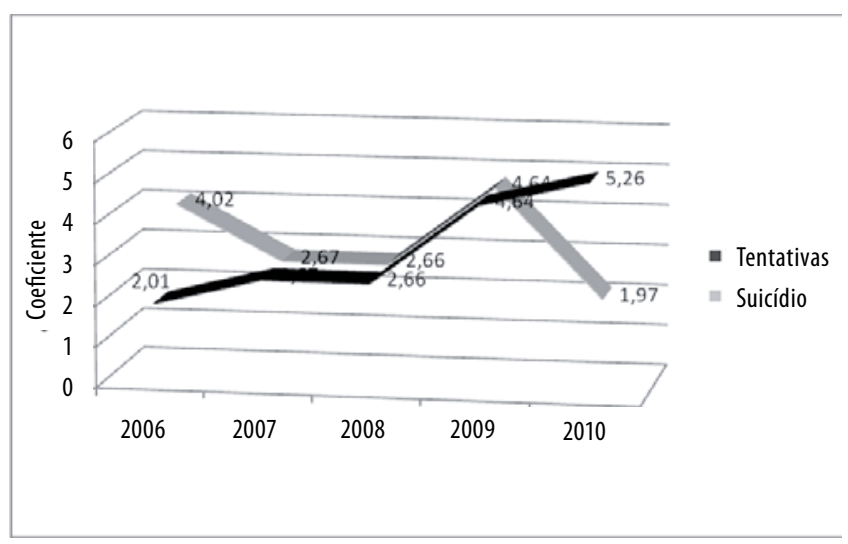

Figura 1. Distribuição do coeficiente de mortalidade por suicídio e tentativas de suicídio no período de 2006 a 2010 (Jequié/BA, 2011).

Dentre as tentativas, verificou-se que a maioria, 61,53\% (n =16), não consumou o ato planejado, geralmente por causa da intervenção do serviço de resgate e/ou de pessoas da comunidade, enquanto 30,76\% ( $n=8$ ) consumaram o ato, mas não foram a óbito. Ressalta-se que, das tentativas, dois casos (7,69\%) não possuíam informações quanto à consumação do ato.

Observou-se predominância de suicídio no sexo masculino, com 87,5\% ( $n=21)$, enquanto nas tentativas predominou o sexo feminino, com 53,4\% ( $n=14)$. Assim, a taxa de mor- 
talidade por suicídio entre os homens foi de 5,71/100.000 e entre as mulheres foi de 0,78/100.000. Já em relação aos casos de tentativas entre os homens, constatou-se uma taxa média de 2,71/100.000 e entre as mulheres, de 3,64/100.000. A proporção de suicídios de mulheres em relação aos homens foi de 1:4. Em comparação, nas tentativas, a proporção de homens em relação às mulheres foi de 1:1,4.

No que se refere à idade, verificou-se que, nos casos de suicídio, houve uma concentração na faixa etária de 40 anos e mais. Já nos casos de tentativas, concentrou-se entre 20 e 39 anos, conforme se observa na tabela 1. Quanto ao intervalo entre idade mínima e máxima, encontrou-se uma variação de 16 a 76 anos para as tentativas e 16 a 77 anos para os casos de suicídio.

Tabela 1. Distribuição dos casos de tentativas de suicídio e suicídio, segundo sexo e idade, no período de 2006 a 2010 (Jequié/BA, 2011)

\begin{tabular}{|c|c|c|c|c|c|c|}
\hline \multirow{3}{*}{$\begin{array}{l}\text { Características segundo } \\
\text { sexo e idade }\end{array}$} & \multicolumn{6}{|c|}{ Evento } \\
\hline & \multicolumn{2}{|c|}{$\begin{array}{l}\text { Tentativas de } \\
\text { suicídio }\end{array}$} & \multicolumn{2}{|c|}{ Suicídio } & \multicolumn{2}{|c|}{ Total } \\
\hline & $\mathrm{N}$ & $\%$ & $\mathrm{~N}$ & $\%$ & $\mathrm{~N}$ & $\%$ \\
\hline \multicolumn{7}{|l|}{ Sexo } \\
\hline Masculino & 10 & 38,46 & 21 & 87,5 & 31 & 62 \\
\hline Feminino & 14 & 53,84 & 3 & 12,5 & 17 & 34 \\
\hline Sem registro & 2 & 7,96 & - & - & 2 & 4 \\
\hline \multicolumn{7}{|l|}{ Faixa etária (em anos) } \\
\hline$<20$ & 2 & 7,69 & 3 & 12,5 & 5 & 10 \\
\hline $20-30$ & 8 & 30,77 & 4 & 16,67 & 12 & 24 \\
\hline $30-40$ & 7 & 26,92 & 4 & 16,67 & 11 & 22 \\
\hline $40-50$ & 6 & 23,08 & 8 & 33,33 & 14 & 28 \\
\hline Acima dos 50 anos & 1 & 3,85 & 5 & 20,83 & 6 & 12 \\
\hline Sem registro & 2 & 7,69 & - & - & 2 & 4 \\
\hline TOTAL & 26 & 100 & 24 & 100 & 50 & 100 \\
\hline
\end{tabular}

No que diz respeito à ocupação das vítimas de suicídio, identificou-se que não havia essa informação no campo correspondente na DO em 25\% $(n=6)$ dos casos. Nas demais, $12,5 \%(n=3)$ eram estudantes, seguidos de profissionais autônomos, carpinteiros, comerciantes e trabalhadores rurais, e cada uma dessas classes correspondia a 8,33\% $(n=2)$. E com menor percentual, verificaram-se as seguintes ocupações: agrônomo, aposentado, do lar, funcionário público, industriário, motorista e pedreiro, com 4,16\% ( $n=1)$ cada.

Em relação ao campo raça/cor da DO, foram encontrados $41,66 \%$ pardos $(n=10), 29,16 \%$ brancos $(n=7), 16,66 \%$ ignorados $(n=4)$ e $12,5 \%$ de cor preta $(n=3)$. Em relação ao estado civil, prevaleceram os solteiros $(41,66 \%, n=10)$, seguidos dos casos ignorados $(41,66 \%, n=10)$ e casados $(16,66 \%$, $n=4)$. A escolaridade apresentou a maior taxa de não preen- chimento com 91,66\% $(n=22)$ e 8,3\% preenchidos com 4 a 7 anos de estudos concluídos $(n=3)$.

Para o suicídio, os meios utilizados foram enforcamento $58,33 \%(n=14)$, seguido de arma de fogo, envenenamento e queda de altura, com o mesmo percentual de $12,5 \%(n=3)$ cada um deles, e queimadura $(4,17 \%, n=1)$. Já nos casos das tentativas, os meios utilizados foram a queda de altura em $69,23 \%(n=18)$ dos casos, seguida de envenenamento, com $23,08 \%(n=6)$ e $7,69 \%(n=2)$ sem informação.

Nos casos de óbitos por suicídio, foram identificadas as seguintes causas de morte de acordo com a Classificação Internacional de Doenças, conforme a tabela 2.

Tabela 2. Distribuição da Classificação Internacional de Doenças (CID-10) nos casos de suicídio, no período de 2006 a 2010 (Jequié/ BA, 2011)

\begin{tabular}{lccc}
\hline \multirow{2}{*}{ Classificação Internacional de Doenças - CID-10 } & \multicolumn{2}{c}{ Suicídio } \\
\cline { 2 - 3 } & N & $\%$ \\
\hline X68 Autointoxicação por pesticidas & 2 & 8,33 \\
X69 Autointoxicação por substâncias não especificadas & 1 & 4,17 \\
X70 Lesão autoprovocada intencionalmente por enforcamento & 14 & 58,3 \\
X74 Lesão autoprovocada intencionalmente por disparos de arma de fogo & 3 & 12,5 \\
X76 Lesão autoprovocada intencionalmente pela fumaça, pelo fogo e por chamas & 1 & 4,17 \\
X80 Lesão autoprovocada intencionalmente por precipitação de lugar elevado & 3 & 12,5 \\
TOTAL & 24 & 100 \\
\hline
\end{tabular}

Os suicídios ocorreram com maior frequência no inverno $(29,17 \%)$ e no verão $(29,17 \%)$, principalmente nos dias de quinta-feira (20,83\%), sexta-feira (16,87\%) e domingo $(16,87 \%)$. Já as tentativas ocorreram, mais frequentemente, no outono $(26,92 \%)$ e primavera $(26,92 \%)$, prevalecendo os dias de sábado $(23,1 \%)$ e terça-feira $(19,23 \%)$.

Em relação aos horários predominantes para o suicídio, não foi possível essa identificação, pois o horário em que ocorreu o evento que precipitou o suicídio não constava no registro da declaração de óbito, mas apenas o horário do próprio óbito, que nem sempre coincide com o horário do ato suicida. Quanto às tentativas, estas acontecem, preferencialmente, nos turnos da manhã $(46,15 \%)$ e noite $(30,77 \%)$.

No município, os suicídios ocorrem com maior frequência em residentes do Centro da cidade (20,83\%) e no bairro do Joaquim Romão (16,67\%). Este último é um dos maiores bairros do município que, por sua grande extensão, abrange tanto o perímetro central da cidade quanto o periférico; enquanto as tentativas foram mais frequentes nos bairros Joaquim Romão (23,08\%) e Curral Novo (19,23\%), sendo este último um bairro periférico.

Tal bairro possui uma característica geográfica que merece destaque no estudo, uma vez que a existência de uma elevada formação rochosa denominada Pedra do Curral Novo tem favorecido a acessibilidade para tentativas de suicídio. 
Nos casos de suicídio, verificou-se uma relação entre meio utilizado e local da ocorrência. Dentre os casos, 83,33\% ( $n=20)$ ocorreram no domicílio, estando mais relacionados a enforcamentos (65\%), lesões por armas de fogo (15\%), envenenamentos (10\%), queimaduras (5\%) e queda de altura (5\%). Quanto aos demais locais, 8,33\% $(n=2)$ dos casos de suicídio ocorreram em decorrência da queda de altura, em que foi utilizada a formação rochosa existente no município e 8,33\% ( $n=2)$ em local não informado, sendo um caso de envenenamento e outro de enforcamento.

Nas tentativas, a relação verificada decorreu entre o meio utilizado e o local de residência. Houve predomínio da precipitação de altura (69,23\%), estando estas relacionadas com os ambientes próximos ao local de residência, como pontes, viaduto, telhados, sacadas e mais destacadamente a Pedra do Curral Novo (61,11\%).

Nota-se que, apesar da tendência ascendente nos casos de tentativa de suicídio, com variação de 2,01/100.000 habitantes em 2006 para 5,26/100.000 habitantes em 2010, os dados de internação referente ao mesmo período apresentam certa linearidade. As taxas anuais foram, respectivamente, 0,67, 1,34, 0,66, 0,0 e 0,65.

\section{DISCUSSÃO}

Os resultados do estudo mostram que a taxa de mortalidade por suicídio no município apresenta oscilações, variando de 4,02 em 2006 a 1,97 em 2010, com a maior taxa no período de 4,64 em 2009 e taxa média de 3,19/100.000 habitantes. No âmbito nacional, outros estudos apresentam a taxa geral de mortalidade por suicídio, no Brasil, estimada entre 4,1 e 5,7/100.000 12,18-20, e, no Nordeste, essa taxa varia entre 2,4 e 3,1 suicídios por $100 \mathrm{mil} /$ habitantes $\mathrm{s}^{12,20}$.

Assim, acompanhando as estimativas nacionais, o município apresenta uma taxa média de mortalidade baixa, contudo, de acordo com o perfil epidemiológico da região Nordeste, essa taxa no município apresenta-se no limiar máximo para a região, constituindo-se em fator relevante para o planejamento das políticas públicas e gestão da saúde em nível local.

Identificou-se que a tentativa de suicídio encontra-se em ascensão no período estudado, constituindo-se em risco para o aumento da mortalidade por suicídio. As taxas de tentativas mais elevadas que as de suicídios convergem com outros estudos ${ }^{19,21}$, estimando-se que as tentativas de suicídio sejam 20 vezes mais frequentes do que os suicídios ${ }^{19}$.

Apesar de no Brasil os óbitos associados ao suicídio constarem nos bancos de dados do SIM, no que se refere às informações sobre as tentativas de suicídio não há registro ou notificação sistemática nos serviços de saúde, fazendo-se necessário consultar informações em outros serviços, como a Polícia Militar ${ }^{21}$ e, neste estudo, o Corpo de Bombeiros.
Mesmo nos casos de suicídio, em que há um sistema de informação que disponibiliza dados sobre o evento, observam-se falhas no preenchimento das DOs e lacunas na alimentação do sistema. Esses fatores geram subnotificação e/ ou discrepâncias entre os dados sobre suicídios nas pesquisas e aqueles disponibilizados no DATASUS 1,19,20. Inconsistências de dados foram identificadas neste estudo ao comparar o número de casos de suicídio registrados no SIM/DATASUS de domínio público com os dados do mesmo período, obtidos diretamente das DOs na 13a DIRES, onde foi encontrado um número menor de casos, semelhante ao que foi identificado no estudo de Parente et al. ${ }^{20}$.

Quanto ao preenchimento das DOs, verificou-se incompletude de modo geral e, principalmente, nas variáveis raça/ cor (16,66\% ignorados), estado civil (41,66\% ignorados) e escolaridade (91,66\% ignorados), que apresentaram baixo índice de completude como também foi observado em outro estudo'. As duas últimas variáveis apresentaram, respectivamente, o índice de completude ruim (não completude de $20 \%$ a $50 \%$ ) e muito ruim (não completude acima de $50 \%$ ), segundo o escore proposto por Romero e Cunha ${ }^{22}$.

Alguns estudos destacam o suicídio como a mais passível de subestimação entre as causas externas ${ }^{1,23}$ de mortalidade, relacionando-se a vários motivos (religiosos, sociais, culturais, políticos, econômicos etc.), os quais geram omissão de informações e subnotificação ${ }^{1,15,16}$. O suicídio apresenta um dos piores índices de preenchimento das DOs, de acordo com o estudo de Macente e Zandonade', que realizaram uma avaliação da completude do sistema de informação sobre mortalidade por suicídio.

No que se refere ao perfil dos casos de suicídio e de tentativas, verificaram-se significativas diferenças, principalmente em relação às características sexo, idade e meios utilizados, que convergem com os resultados encontrados por Macente et al. ${ }^{21}$. Quanto ao sexo, os resultados estão em conformidade com a produção científica, ocorrendo o predomínio de suicídio no sexo masculino 5,12,19-21,24-27, com proporção de quatro casos masculinos para um feminino, corroborando o estudo de Viana et al. ${ }^{24}$, enquanto as tentativas ocorrem mais destacadamente entre o sexo feminino ${ }^{13,19,21,28}$.

Em relação à idade, as tentativas são mais comuns entre os jovens, enquanto o suicídio é mais frequente entre a população acima de 40 anos, também identificado em outros estudos 9,12,13,19,21-26,28. Isso reflete a influência que os fatores socioeconômicos e psicossociais ${ }^{19}$ relacionados às fases do ciclo vital exercem sobre a tentativa de suicídio, mais comum em adultos jovens ${ }^{9,13,28}$, enquanto a consumação com êxito se observa mais nas idades avançadas 12,19,21,24-26.

Nota-se uma relação entre os meios utilizados e o índice de letalidade ${ }^{19}$, tendo em vista que, nos suicídios, o meio mais utilizado foi o enforcamento (58,33\%) $)^{12,19-21,24-27}$, enquanto nas tentativas os meios mais utilizados foram a ameaça de precipitação de altura $(69,23 \%)$ e o envenenamento 
(23,08\%), aproximando-se de outros estudos com resultados semelhantes ${ }^{12,19,20,24-26,28,}$. A ameaça de precipitação de altura como meio mais utilizado nas tentativas merece destaque, pois $61,11 \%$ ocorreram em uma elevação rochosa no município, sugerindo que os fatores geográficos podem favorecer a escolha do método.

Ainda em relação às tentativas na elevação rochosa, observou-se que, nos casos em que houve intervenção da equipe de resgate, foi possível evitar a precipitação, por causa do treinamento específico que o Corpo de Bombeiros possui para esse tipo de intervenção. Isso reforça a necessidade de que as equipes do SAMU também sejam qualificadas para o atendimento às tentativas de suicídio em lugares de risco. Verificou-se que nas tentativas as intervenções foram realizadas pela equipe de bombeiros e população local (religiosos, familiares, vizinhos, amigos e pessoas que passavam no local). Logo, o papel das equipes de saúde restringiu-se à prestação de atendimento nos casos de lesão física em consequência da efetivação do plano suicida.

Os meios utilizados estão associados ao perfil das pessoas que cometem e tentam o suicídio, principalmente ao sexo e idade. A maioria dos que obtêm sucesso é de homens com faixa etária de mais idade ${ }^{19,26}$, logo utilizam meios mais violentos e letais, como enforcamento e disparo de arma de fogo ${ }^{24,25,27}$, enquanto nas tentativas a maioria é de mulheres e jovens ${ }^{26}$, que escolhem métodos mais suaves e lentos, com destaque para o envenenamento e a queda de altura ${ }^{19,21}$. Tais características perpassam os signos da masculinidade e da feminilidade e relacionam-se à produção de sentidos acerca do suicídio e da tentativa, para ambos os sexos ${ }^{21,29}$.

Aos meios utilizados relacionou-se, ainda, o local do evento e residência. Estes, por sua vez, possuem influência, respectivamente, sobre a disponibilidade e a acessibilidade aos meios ${ }^{19}$. Observou-se que $83,33 \%$ dos suicídios ocorreram na residência ${ }^{12,25}$, por meio de enforcamento (65\%), seguido de disparos de arma de fogo, envenenamentos e queda de altura, convergindo com outros estudos ${ }^{12,25}$. Nesse sentido, as equipes de saúde da família e de saúde mental podem ser capacitadas para a identificação das pessoas e grupos vulneráveis ao evento, a fim de orientar as famílias e pessoas que convivem com tais grupos, visando à vigilância e à prevenção do evento ${ }^{14,30}$.

Em relação às tentativas, o meio mais utilizado foi a precipitação de altura $(69,23 \%)^{19,21}$, estando relacionadas aos ambientes próximos da residência, como pontes, viaduto, telhados, sacadas e, mais destacadamente, a Pedra do Curral Novo $(61,11 \%)$. O fato de ocorrerem em locais públicos favorece a intervenção em tempo oportuno, enquanto os casos que ocorrem no domicílio, na maioria das vezes, não são percebidos antes da consumação do ato. Nos casos de tentativa no domicílio, a intervenção ocorre após o dano, como nas tentativas por ingestão de substâncias químicas e medicamentos $(30,77 \%)$.
No que se refere aos dias da semana, constatou-se que, no suicídio, houve maior concentração na quinta-feira, seguida da sexta-feira e do domingo, convergindo com outro estudo em relação à sexta-feira ${ }^{21}$. Nas tentativas, essa concentração ocorreu no sábado, seguido da terça-feira. Os turnos de maior ocorrência das tentativas são as manhãs e as noites, percebendo-se que no período da manhã ocorreram, principalmente, as tentativas por precipitação de altura.

Quanto à morbidade por tentativas de suicídio, ao investigar os registros do $\mathrm{SIH}$, encontrou-se discrepância em relação à característica ascendente das tentativas. Apenas os casos mais graves levam à internação hospitalar, sendo a maioria dos casos atendida nas emergências, o que acaba não gerando $\mathrm{AlHs}^{21}$.

Assim, os dados de internação obtidos no Sistema de Informação Hospitalar, referentes a 2009, destoam dos dados de mortalidade e tentativas encontrados no estudo, pois esse foi o ano que apresentou maior taxa de mortalidade $(4,64 / 100.000$ habitantes) e a segunda maior taxa de tentativas, enquanto as internações registradas decorrentes das tentativas de suicídio, no ano de 2009, apresentaram taxa zero.

Dentre as limitações do estudo, destaca-se a escassez de dados oficiais sobre tentativas de suicídio, que levou à restrição da utilização aos casos atendidos pelo Corpo de Bombeiros. Ao observar que muitas intervenções são feitas por populares, amigos e familiares das vítimas, ou pelas equipes de Saúde Mental, Saúde da Família e Serviço Pré-hospitalar, considera-se uma provável subestimação de casos.

Outra limitação diz respeito à impossibilidade de verificação das reincidências dos casos, dado o caráter transversal do estudo. Logo, faz-se necessário desenvolver pesquisas, com maior amplitude e utilização de fontes de dados diversas, que apresentem uma caracterização mais fidedigna do perfil de tentativas, bem como a correlação destas com transtornos psiquiátricos e uso de substâncias psicoativas.

Além disso, percebe-se grande lacuna de conhecimento no que se refere aos estudos sobre suicídio e tentativas de suicídio na região Nordeste, bem como pesquisas em municípios de pequeno e médio porte. Assim, este estudo visa contribuir com a construção de conhecimento nessa perspectiva e também apontar para a necessidade de novos estudos na área.

\section{CONCLUSÃO}

Os resultados apontam para a diferenciação entre as características do perfil das tentativas de suicídio e mortalidade por suicídio, especialmente segundo o sexo, faixa etária e meios utilizados. As tentativas são mais frequentes entre as mulheres jovens, por meio da precipitação de altura ou outros métodos que não levam à morte imediata, sendo a maioria em 
locais públicos, onde a atuação dos serviços tem conseguido diminuir a consumação do ato. Quanto aos suicídios, acontecem entre homens acima de 40 anos, por enforcamento, escolhendo o domicílio como local para a sua consumação, o que dificulta a intervenção.

Os dados de mortalidade por suicídio entre os anos de 2006 e 2009, no município, encontram-se em consonância com as estatísticas da região Nordeste e abaixo das taxas nacionais de suicídio. Quanto aos dados encontrados, verificou-se a incompletude no preenchimento das DOs, principalmente em relação à escolaridade, estado civil e raça/cor. Além disso, foi identificada a necessidade da melhoria dos registros oficiais, dos serviços de saúde, especialmente, em relação às tentativas.

\section{REFERÊNCIAS}

1. Macente LB, Zandonade E. Avaliação da completude do sistema de informação sobre mortalidade por suicídio na região Sudeste, Brasil, no período de 1996 a 2007. J Bras Psiquiatr. 2010;59(3):173-81.

2. Kapczinski F, Quevedo J, Schitt R, Chachamovich E. Emergências psiquiátricas. Porto Alegre: Artmed; 2001

3. Brasil. Ministério da Saúde. Temático Prevenção de Violência e Cultura da Paz II - Painel de indicadores do SUS n0 5. Brasília: Organização Pan-Americana da Saúde; 2008.

4. Werneck GL, Hasselmann MH, Phebo LB, Vieira DE, Gomes VLO. Tentativas de suicídio em um hospital geral no Rio de Janeiro, Brasil. Cad Saude Publica. 2006;22(10):2201-6.

5. Bahls SC, Botega NJ. Epidemiologia das tentativas de suicídio e dos suicídios. In: Mello MF, Mello AAF, Kohn R. Epidemiologia da saúde mental no Brasil. Porto Alegre: Artmed; 2007. p. 151-71

6. WHO (World Health Organization). Figures and facts about suicide. Geneva; 1999.

7. Baptista MN. Suicídio e depressão - atualizações. Rio de Janeiro: Guanabara Koogan; 2004.

8. Organização Mundial da Saúde (OMS). Relatório sobre a saúde no mundo 2001: saúde mental - nova concepção, nova esperança. Genebra; 2001.

9. Lima DD, Azevedo RCS, Gaspar KC, Silva VF, Mauro MLF, Botega NJ. Tentativa de suicídio entre pacientes com uso nocivo de bebidas alcoólicas internados em hospital geral. J Bras Psiquiatr. 2010;59(3):167-72.

10. Werlang BSG, Botega NJ. Comportamento suicida. Porto Alegre: Artmed; 2004.

11. Meneghel SN, Victora CG, Faria NMX, Carvalho LA, Falk JW. Características epidemiológicas do suicídio no Rio Grande do Sul. Rev Saude Publica. 2004;38(6):804-10.
12. Lovisi GM, Santos AS, Legay L, Abelha L, Valencia E. Análise epidemiológica do suicídio no Brasil entre 1980 e 2006. Rev Bras Psiquiatr. 2009;31(Supl II):86-93.

13. Diehl A, Laranjeira R. Suicide attempts and substance use in an emergency room sample. J Bras Psiquiatr. 2009;58(2):86-91.

14. Brasil: Ministério da Saúde - Manual de Prevenção ao Suicídio dirigido aos profissionais das equipes de saúde mental. 2006. Disponível em: http://bvsms.saude.gov.br/bvs/publicacoes/manual_editoracao.pdf. Acesso em: 15 jun. 2011.

15. Brasil. Ministério da Saúde. Impacto da violência na saúde dos brasileiros. Brasília: Ministério da Saúde; 2005.

16. Volpe FM, Corrêa H, Barrero SP. Epidemiologia do suicídio. In: Corrêa H, Barrero SP. Suicídio: uma morte evitável. São Paulo: Atheneu; 2006. p. 10-27.

17. IBGE. Instituto Brasileiro de Geografia e Estatística. Contagem da população 2010. Disponível em: http://www.ibge.gov.br/cidadesat/topwindow.htm?1. Acesso em: 16 jun. 2011.

18. WHO (World Health Organization). Prevention of suicidal behaviors: a task for all. In: Mental and Behavioral Disorders. Department of Mental Health, World Health Organization, Geneva; 2000.

19. Prieto D, Tavares M. Fatores de risco para suicídio e tentativa de suicídio: incidência, eventos estressores e transtornos mentais. J Bras Psiquiatr. 2005;54(2):146-54.

20. Parente ACM, Soares RB, Araújo ARF, Cavalcante IS, Monteiro CFS. Caracterização dos casos de suicídio em uma capital do Nordeste brasileiro. Rev Bras Enferm. 2007;60(4):377-81.

21. Macente LB, Santos EG, Zandonade E. Tentativas de suicídio e suicídio em município de cultura pomerana no interior do estado do Espírito Santo. J Bras Psiquiatr. 2009;58(4):238-44.

22. Romero DE, Cunha CB. Avaliação da qualidade das variáveis socioeconômicas e demográficas dos óbitos de crianças menores de 1 ano registrados no Sistema de Informações sobre Mortalidade do Brasil (1996/2001). Cad Saude Publica. 2006;22(3):673-84.

23. Mello Jorge MHP. Mortalidade por causas violentas no município de São Paulo [tese]. São Paulo: Faculdade de Saúde Pública da Universidade de São Paulo; 1995.

24. Viana GN, Zenkner FM, Sakae TM, Escobar, BT. Prevalência de suicídio no sul do Brasil, 2001-2005. J Bras Psiquiatr. 2008;57(1):38-43.

25. Marín-León L, Barros MB. Mortes por suicídio: diferenças de gênero e nível socioeconômico. Rev Saude Publica. 2003;37(3):357-63.

26. Schmitt R, Lang MG, Quevedo J, Colombo T. Perfil epidemiológico do suicídio no extremo oeste do estado de Santa Catarina, Brasil. Rev Psiquiatr RS. 2008;30(2):115-23.

27. Baptista MN, Borges A. Suicídio: aspectos epidemiológicos em Limeira e adjacências no período de 1998 a 2002. Estud Psicol (Campinas). 2005;22(4):425-43.

28. Botega NJ, Marín-León L, Oliveira HB, Barros MB, Silva VF, Dalgalarrondo P. Prevalências de ideação, plano e tentativa de suicídio: um inquérito de base populacional em Campinas, São Paulo, Brasil. Cad Saude Publica. 2009;25(12):2632-8.

29. Lopes FH. Medicina, educação e gênero: as diferenciações sexuais do suicídio nos discursos médicos do século XIX. Educ Rev [online]. 2007;29:241-57.

30. Botega NJ. Suicídio: saindo da sombra em direção a um Plano Nacional de Prevenção. Rev Bras Psiquiatr. 2007;29(1):7-8. 